\title{
Enfermedad de Bowen perianal: presentación de
} dos casos

Perianal Bowen's disease: two cases report

\section{Óscar Jairo Valencia Ocampo', Marta Cecilia Sierra Sierra², Juan Guillermo Hoyos Gaviria ${ }^{3}$}

1. Dermatólogo, Profesor. Centro de Investigaciones Dermatológicas, CIDERM, Unidad de Fotodermatología sede IPS Universitaria, Sección de Dermatología, Facultad de Medicina, Universidad de Antioquia

2. Dermatóloga, Profesora. Sección de Dermatología, Fototerapia Sede San Vicente Fundación Hospital Universitario, Facultad de Medicina, Universidad de Antioquia

3. Dermatólogo, Profesor. Sección de Dermatología, Facultad de Medicina, Universidad de Antioquia

\section{RESUMEN}

El compromiso de la región perianal por enfermedad de Bowen es poco frecuente. $\mathrm{Su}$ etiología se ha relacionado con la infección por los virus del papiloma humano. La enfermedad de Bowen perianal puede hallarse de forma incidental y los síntomas que produce son inespecíficos. Puede expresarse como placas eritematosas, blanquecinas y, ocasionalmente, pigmentadas, y simular las dermatitis eccematosas o un liquen simple crónico. La evolución es lenta y entre el 2\% y el $6 \%$ de los casos pueden progresar a un carcinoma escamocelular invasor. El tratamiento puede ser quirúrgico o con modalidades no quirúrgicas, entre las cuales se cuenta con la terapia fotodinámica, considerada como un tratamiento de primera línea para la enfermedad de Bowen por su seguridad y efectividad a corto plazo; sin embargo, parece que la recurrencia a largo plazo es frecuente. Se presentan dos casos de enfermedad de Bowen perianal. El primer caso representa la forma pigmentada, una variante de presentación clínica poco frecuente, tratado con cirugía local amplia. El segundo caso recibió tratamiento conservador con terapia fotodinámica, con una respuesta satisfactoria a corto plazo.

PALABRAS CLAVE: enfermedad de Bowen, neoplasias del ano, enfermedades del ano, carcinoma de células escamosas, carcinoma in situ.

\section{SUMMARY}

The compromise of the perianal region due to Bowen's disease is rare, its etiology has been related to human papillomavirus infection. Perianal Bowen's disease can be found incidentally and the symptoms they produce are nonspecific, it can be expressed as erythematous, whitish and occasionally pigmented plaques, it can resemble as eczematous dermatitis or lichen simplex chronicus. Evolution is slow, between $2 \%$ and $6 \%$ of cases can progress to an invasive squamous cell carcinoma. The treatment can be surgical or not. Among non-surgical treatments include Photodynamic therapy, which is considered a first line treatment, it is safe and effective in the short term, however, it seems
Correspondencia:

Óscar Jairo Valencia Ocampo

Email:

oscar.valencia@udea.edu.co

Recibido: 05/02/18

Aceptado: 05/05/18

Conflictos de interés:

No se reportan conflictos de interés.

\section{Financiación:}

Ninguna. 
that recurrences are frequent in the long term. Two cases of perianal Bowen disease are presented. The first case represents the pigmented form, a variant of uncommon clinical presentation, treated with extensive local surgery. The second case received a conservative treatment with photodynamic therapy with a satisfactory response in the short term.

KEY WORDS: Bowen's disease, anus neoplasms, anus diseases, carcinoma squamous cell, carcinoma in situ.

\section{CASO CLÍNICO 1}

Se trata de una mujer de 75 años, sin antecedentes patológicos de importancia, que consultó por prurito pe- rianal de varios años de evolución, tratada previamente con esteroides tópicos, protectores cutáneos con óxido de zinc al 20\% en crema sin mejoría; la paciente no refería otros síntomas asociados. En el examen físico se observaron cinco placas de $2 \mathrm{~cm}$ a $6 \mathrm{~cm}$ en sus diámetros mayores, color marrón claro y oscuro, de contornos irregulares y bien definidos, con algunas áreas hiperqueratósicas, otras erosionadas y con fisuras, localizadas en la región perianal (figura 1). En el examen histopatológico se describieron cambios de hiperplasia epidérmica con queratinocitos atípicos, que confirmaron el diagnóstico de enfermedad de Bowen. Se realizó tratamiento quirúrgico con resección local amplia y márgenes libres de enfermedad de un centímetro. El control histopatológico posquirúrgico fue satisfactorio.

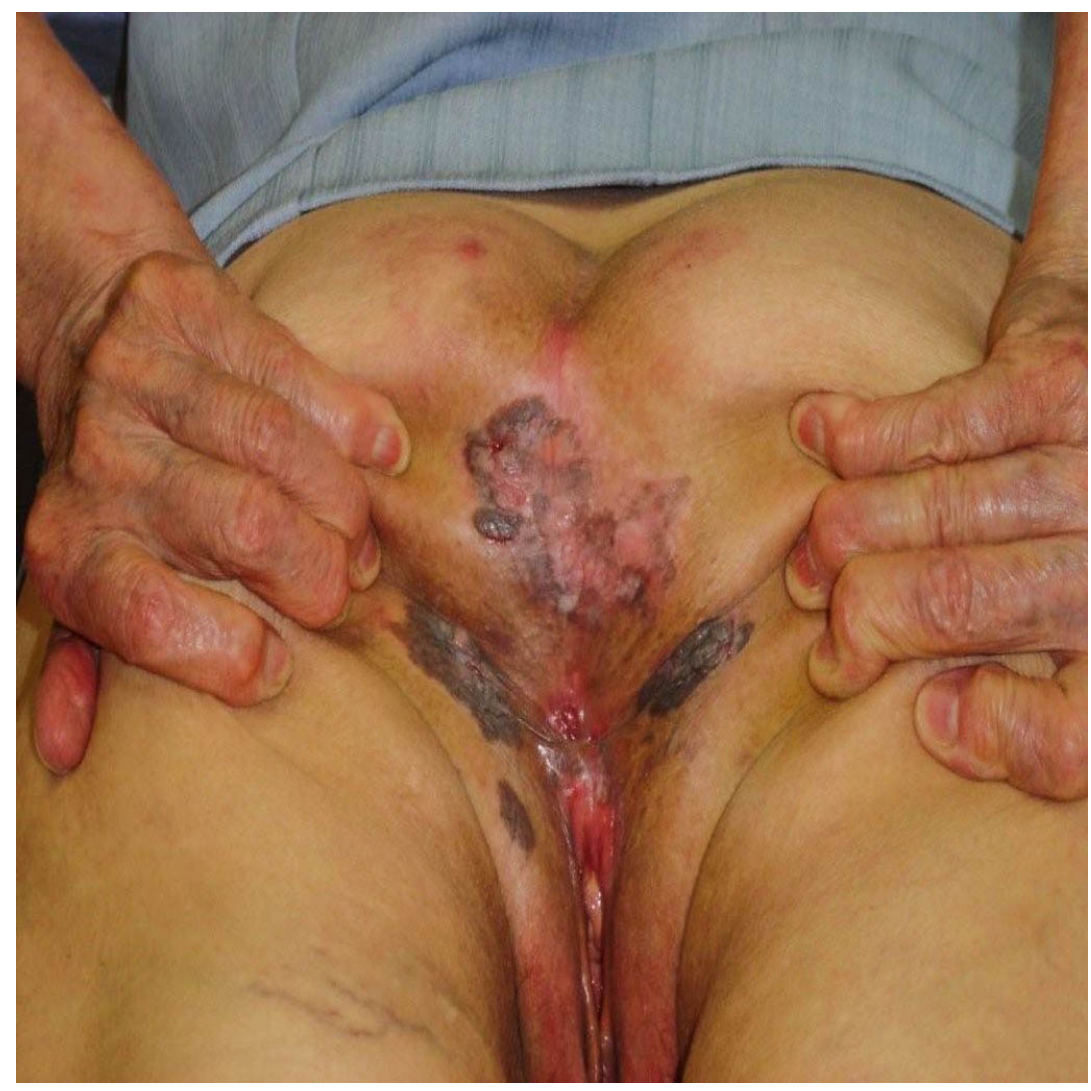

Figura 1. Numerosas placas irregulares y bien definidas, marrón claras y oscuras, con áreas hiperqueratósicas, erosiones y fisuras. Localizadas en la región perianal. 


\section{CASO CLIINICO 2}

Mujer de 70 años, que consultó por una lesión dolorosa perianal de 6 años de evolución. Recibió manejo tópico con hidrocortisona al 1\% y clotrimazol al 1\% en cremas, sin mejoría. En el examen físico se observó una placa localizada en la región perianal superior izquierda, entre las 9 y 2 del reloj, que medía $12 \mathrm{~cm}$ por $11 \mathrm{~cm}$ en sus longitudes mayores, de forma trilobular, con bordes bien definidos, de un color eritematoso rosado con áreas blanquecinas vegetantes y áreas erosionadas, con una secreción serosa escasa (figura 2). En el examen histopatológico se describieron cambios de hiperplasia epidérmica con queratinocitos atípicos, que confirmaron el diagnóstico de enfermedad de
Bowen. Recibió tres sesiones de terapia fotodinámica con un intervalo de 25 días entre los tratamientos, se usó aminolevulinato de metilo en $160 \mathrm{mg} / \mathrm{g}$ en crema, la cual se aplicó por un período de tres horas, con posterior exposición a luz roja de $635 \mathrm{~nm}$, emitida por una lámpara LED Aktilite CL128, con una fluencia de $37 \mathrm{~J} / \mathrm{cm}^{2}$, a una distancia de la piel de entre $5 \mathrm{~cm}$ a 8 cm y por un tiempo de 8 minutos. La paciente presentó respuesta satisfactoria con remisión de las lesiones.

No se realizó un seguimiento de la evolución de los casos luego de los tratamientos realizados.

Los hallazgos histopatológicos en ambos casos fueron similares (figuras 3 y 4).

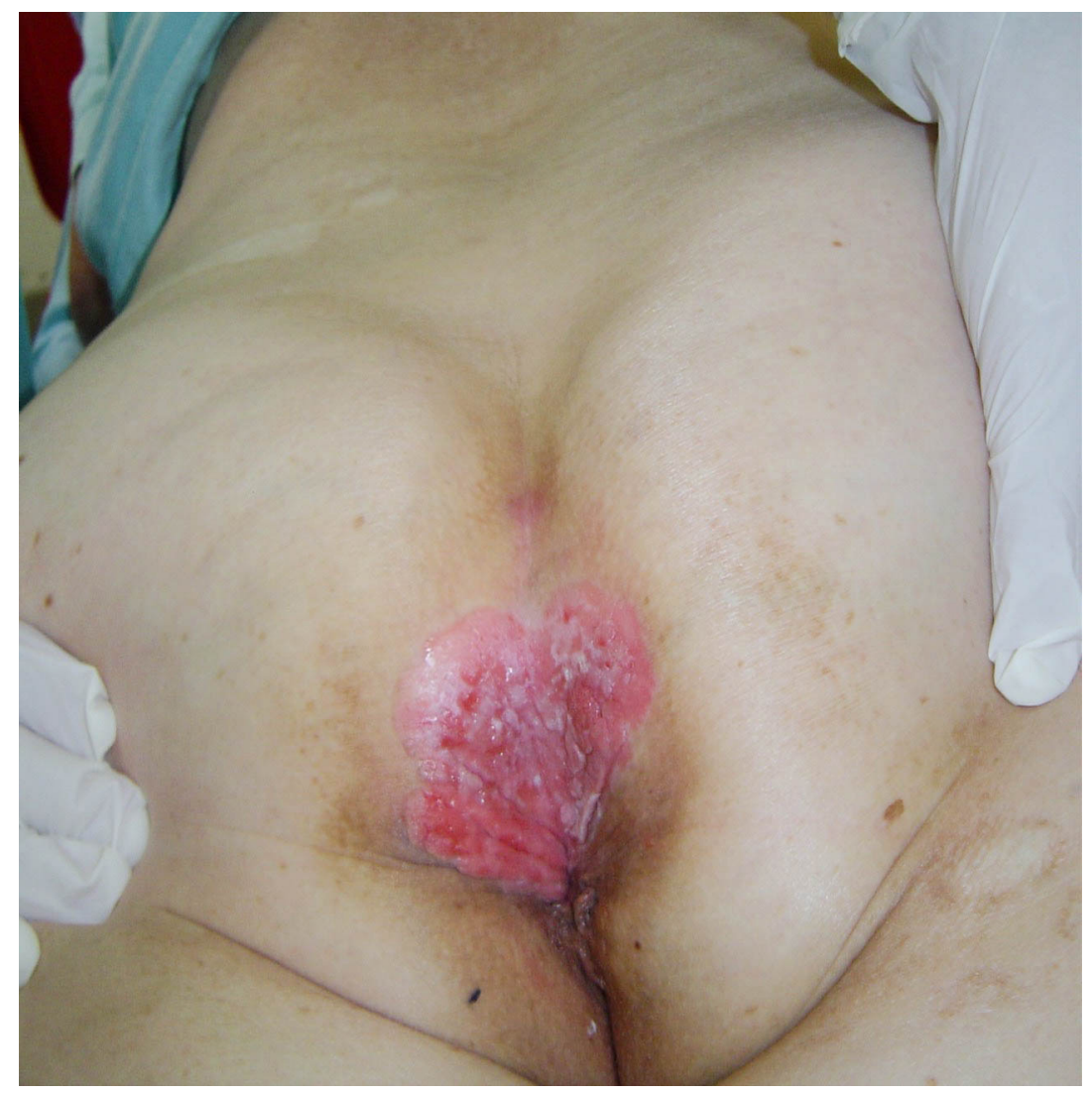

Figura 2. Placa rosada bien definida de $12 \mathrm{~cm}$ por $11 \mathrm{~cm}$, con áreas blanquecinas vegetantes y erosionadas, y secreción serosa escasa. Localizada en la región perianal superior izquierda. 


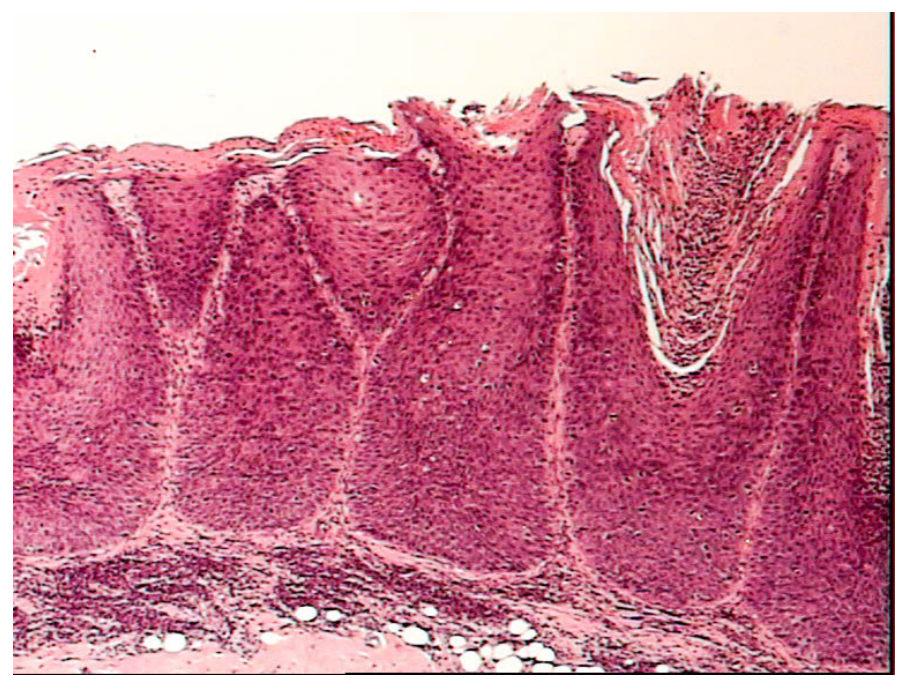

Figura 3. Epidermis con gran acantosis regular, despolarización y pleomorfismo celular que ocupa todo el espesor, dermis superior con infiltrado inflamatorio crónico en banda. Mediano aumento. $10 X$.

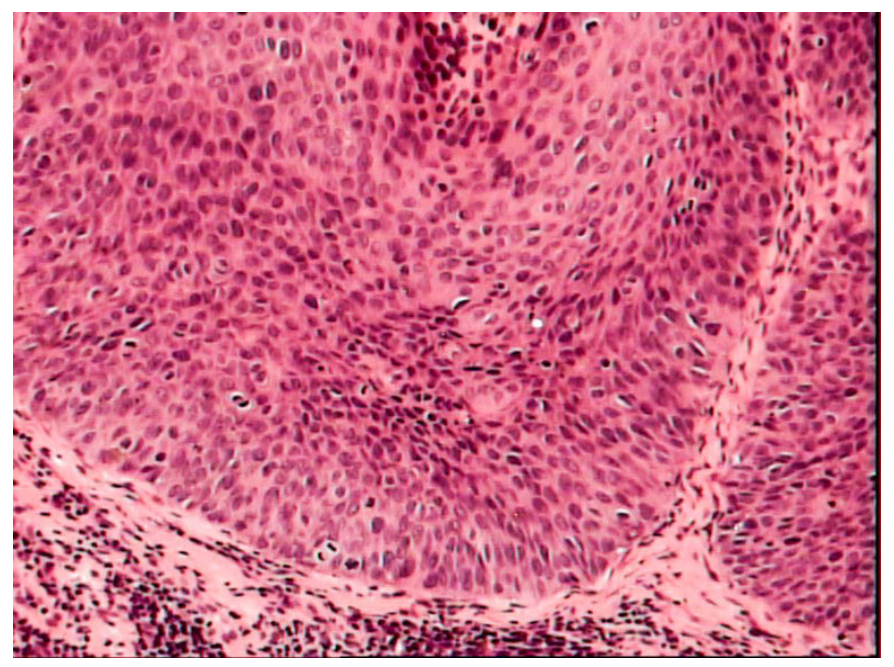

Figura 4. Detalle de la imagen anterior. Gran aumento. 40X. 


\section{COMENTARIO}

La enfermedad de Bowen es un carcinoma escamoso intraepitelial, que fue descrito por primera vez por John T. Bowen, en 1912. En la etiopatogenia se ha descrito su relación con factores como la radiación ionizante, la exposición solar, la exposición al arsénico, la inmunosupresión, la infección por virus del papiloma humano (VPH), los factores genéticos, los traumatismos y los carcinógenos químicos ${ }^{(1)}$. La afectación de la zona perianal es poco frecuente y se presenta más en mujeres mayores de 50 años que en hombres. Se ha asociado con la infección por los VPH de los serotipos 6, 11,16 y 18 de la región anogenital. El riesgo de progresión a carcinoma escamocelular invasor es del $2 \%$ al $6 \%$ y se relaciona con la infección por los serotipos de VPH 16 y 18; el riesgo parece mayor en pacientes con infección por VIH y conteos de CD4 menores de $200^{(2,3)}$. El uso de las vacunas disponibles para VPH puede prevenir el desarrollo de la enfermedad de Bowen perianal.

La forma pigmentada de enfermedad de Bowen perianal es rara, corresponde a menos del $2 \%$ de los casos, se desconoce el mecanismo de la pigmentación y no se han demostrado diferencias en su comportamiento biológico frente a las formas no pigmentadas ${ }^{(4)}$.

La enfermedad de Bowen perianal a menudo se diagnostica incidentalmente durante el examen histopatológico del tejido perianal eliminado durante otro procedimiento (por ejemplo, hemorroidectomía) o durante la biopsia de la piel perianal para quejas inespecíficas. Los pacientes pueden referir prurito, ardor, dolor local, sensación de masa y sangrado. La evolución es lenta y progresiva; el diagnóstico puede retardarse varios años. En una serie de 25 casos tratados con resección quirúrgica en Estados Unidos, se reportó una duración promedio de los síntomas antes del diagnós-

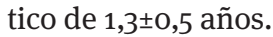

La enfermedad de Bowen perianal se caracteriza clínicamente por la presencia de una o varias placas, que miden de pocos milímetros a varios centímetros; la mayoría pueden ser eritematosas o blanquecinas y en ocasiones de color marrón claro u oscuro, su superficie puede ser lisa, aterciopelada o verrucosa y se describe que pueden semejar un eczema subagudo o un liquen simple crónico ${ }^{(4,5)}$.

La histología de la enfermedad de Bowen es similar en la zona perianal a lo descrito en otras zonas anatómicas. Se reportan anormalidades en el patrón de queratinización y de la arquitectura de la epidermis con hiperplasia epidérmica y elongación de la red de crestas, con queratinocitos atípicos, los cuales presentan alteración de la morfología normal, ausencia de maduración y de mitosis, que afectan a todo el espesor de la epidermis ${ }^{(6)}$.

Los diagnósticos diferenciales más importantes son la enfermedad de Paget (adenocarcinoma intraepitelial), la papulosis bowenoide y el melanoma ${ }^{(7)}$.

Para el tratamiento de la enfermedad de Bowen se describen las intervenciones quirúrgicas como la resección local amplia con márgenes que se extienden a $1 \mathrm{~cm}$ de la lesión o la cirugía micrográfica de Mohs, además de los tratamientos destructivos, como la cauterización con curetaje o la criocirugía; y, finalmente, el uso de otras técnicas como la terapia tópica con cremas de imiquimod al 5\% o 5 fluoruracilo al 5\%, la terapia fotodinámica, el láser de argón y la radioterapia. Para la elección de la intervención se recomienda considerar las características individuales del paciente, como la edad, la ubicación, el tamaño de las lesiones y las comorbilidades ${ }^{(8,9)}$.

La terapia fotodinámica (TFD) parece un método de tratamiento efectivo y seguro. En los últimos años, se ha considerado como primera línea de tratamiento para la enfermedad de Bowen, con respuestas clínicas que varían entre un 52\% y un 100\%. La TFD puede realizarse con ácido 5-aminolevulínico (ALA) o metil-aminolevulinato (MAL). Ambas modalidades de tratamiento son bien toleradas y cuando se comparan con la crioterapia, los resultados cosméticos son mejores debido a que no dejan cicatriz. No se encontraron ensayos aleatorios controlados que compararan las modalidades de TFD-ALA y TFD-MAL. En pacientes con enfermedad de Bowen tratados con TFD-ALA no existen diferencias significativas en la respuesta clínica por aclaramiento de las lesiones cuando se tratan con luz roja o luz verde; sin embargo, parece que la recurrencia de las lesiones es un poco menor con la TFD-ALA con luz roja ${ }^{(10)}$. Estudios recientes sobre la efectividad a largo plazo de la TFD-MAL han demostrado un riesgo de recurrencia luego del tratamiento de alrededor del 30\% a 5 años, en especial para lesiones mayores de $300 \mathrm{~mm}^{2}$ o mayores de $21 \mathrm{~mm}$ de diámetro. Por lo anterior, se recomienda un seguimiento estrecho de los casos de enfermedad de Bowen tratados con esta modalidad terapéutica por un período mínimo de 5 años ${ }^{(11)}$.

Hay controversia sobre la relación de la enfermedad de Bowen perianal con la ocurrencia de otros carcinomas 
cutáneos o viscerales. Aunque algunos autores indican que existe una relación, esta parece ser baja; los carcinomas vulvares son los más reportados ${ }^{5}$. Se recomienda el seguimiento a largo plazo de estos pacientes para la vigilancia de la aparición de segundas neoplasias. Los expertos recomiendan controles médicos una o dos veces por año con anoscopia y rectosigmoidoscopia. La realización de colonoscopia se hará según el riesgo individual de carcinoma colorrectal, cada 3 a 5 años.

\section{CONCLUSIÓN}

La afectación perianal por enfermedad de Bowen es poco frecuente, puede ser un hallazgo casual en las formas asintomáticas y debería tenerse en cuenta dentro de los diagnósticos diferenciales de las dermatitis en esta localización. Se reportaron dos casos clínicos que representan las formas de presentación con lesiones tipo placas pigmentadas y eritematodescamativas, tratados con cirugía local amplia y con TFD-MAL, respectivamente. Luego del tratamiento de pacientes con enfermedad de Bowen perianal, se recomienda realizar un seguimiento por un mínimo de 5 años, debido al riesgo de recurrencias locales y de segundas neoplasias.

\section{REFERENCIAS}

1. Drake AL, Walling HW. Variations in presentation of squamous cell carcinoma in situ (Bowen's disease) in immunocompromised patients. J Am Acad Dermatol. 2008;59(1):68-71.

2. Sarmiento JM, Wolff BG, Burgart LJ, Frizelle FA, Ilstrup DM. Perianal Bowen's disease associated tumors, human papillomavirus, surgery and others controversies. Dis Colon Rectum. 1997;40(8):912-8.

3. Beck DE, Fazio VW, Jagelman DG, Lavery IC. Perianal Bowen's disease. Dis Colon Rectum. 1988;31(6):419-22.

4. Papageorgiou PP, Koumarianou AA, Chu AC. Pigmented Bowen's disease. Br J Dermatol. 1998;138(3):515-8.

5. Margenthaler JA, Dietz DW, Mutch MG, Birnbaum EH, Kodner IJ, Fleshman JW. Outcomes, risk of other malignancies, and need for formal mapping procedures in patients with perianal Bowen's disease. Dis Colon Rectum. 2004;47(10):1655-60.

6. Zhan W, Liao X, Tian T, Zhang Y, Li P, Wu XP, et al. Perianal multiple Bowen's disease: a case report. Int J Clin Exp Pathol. 2015;8(11):15039-41.
7. Krishnan R, Lewis A, Orengo I, Rosen T. Pigmented Bowen's disease (squamous cell carcinoma in situ): a mimic of malignant melanoma. Dermatol Surg. 2001;27(7):673-4.

8. Runfola MA, Weber TK, Rodriguez-Bigas MA, Dougherty TJ, Petrelli NJ. Photodynamic therapy for residual neoplasms of the perianal skin. Dis Colon Rectum. 2000;43(4):499-502.

9. Jansen MH, Appelen D, Nelemans PJ, Winnepenninckx VJ, Kelleners-Smeets NWJ, Mosterd K. Bowen's Disease: Long-term Results of Treatment with 5-Fluorouracil Cream, Photodynamic Therapy or Surgical Excision. Acta Derm Venereol. 2018;98(1):114-115.

10. Bath-Hextall FJ, Matin RN, Wilkinson D, LeonardiBee J. Interventions for cutaneous Bowen's disease. Cochrane Database Syst Rev. 2013;(6):CDoo7281.

11. Aguilar-Bernier M, Rodríguez-Barón D, RivasRuiz F, Segura-Palacios JM, de Troya Martín M. Long-term efficacy of photodynamic therapy with methyl aminolevulinate in treating Bowen's disease in clinical practice: a retrospective cohort study (2006-2017). Photodermatol Photoimmunol Photomed. 2019;35(4):208-213. 